\title{
Strong Bounds on Onsager Coefficients and Efficiency for Three-Terminal Thermoelectric Transport in a Magnetic Field
}

\author{
Kay Brandner, ${ }^{1}$ Keiji Saito, ${ }^{2}$ and Udo Seifert ${ }^{1}$ \\ ${ }^{1}$ II. Institut für Theoretischen Physik, Universität Stuttgart, 70550 Stuttgart, Germany \\ ${ }^{2}$ Department of Physics, Keio University, 3-14-1 Hiyoshi, Kohoku-ku, Yokohama 223-8522, Japan
}

(Received 12 November 2012; published 11 February 2013)

\begin{abstract}
For thermoelectric transport in the presence of a magnetic field that breaks time-reversal symmetry, a strong bound on the Onsager coefficients is derived within a general setup using three terminals. Asymmetric Onsager coefficients lead to a maximum efficiency substantially smaller than the Carnot efficiency reaching only $\eta_{C} / 4$ in the limit of strong asymmetry. Related bounds are derived for efficiency at maximum power, which can become larger than the Curzon-Ahlborn value $\eta_{C} / 2$, and for a cooling device. Our approach reveals that in the presence of reversible currents the standard analysis based on the positivity of entropy production is incomplete without considering the role of current conservation explicitly.
\end{abstract}

DOI: 10.1103/PhysRevLett.110.070603

PACS numbers: 05.70.Ln, 72.15.Jf

The Onsager reciprocity theorem derived more than eighty years ago is arguably the most important constraint for the phenomenological framework of linear irreversible thermodynamics [1]. In its original form it states that the linear kinetic coefficient $L_{i k}(\mathbf{B})$, which relates the affinity $\mathcal{F}_{k}$ to the flux $J_{i}$ is equal to the reciprocal coefficient $L_{k i}(-\mathbf{B})$ provided both $i$ and $k$ refer to quantities with even signature under time reversal [2]. The constant magnetic field $\mathbf{B}$ breaks the time-reversal symmetry. A generalization which includes variables with odd signature was given by Casimir in 1948 [3]. Ever since, the reciprocal relations have turned out to be extremely useful for a plethora of applications. A prominent one is the unified theory of thermoelectricity which allows one to treat the various thermoelectric effects such as the Peltier, the Seebeck, or the Thomson effect on an equal footing thus revealing their interdependencies $[2,4]$.

It is well known that properly designed energy filters allow a substantial enhancement of the efficiency of thermoelectric devices [5]. Linke and co-workers [6,7] showed that, in principle, even Carnot efficiency is attainable. Despite these promising theoretical results, the actual performance of the present devices is notoriously much more modest, which keeps the search for better thermoelectric materials a very active and increasingly important research field as reviewed in Refs. [8-11]. Recently, in an intriguing paper, Benenti et al. pointed out that the presence of a magnetic field could, in principle, enhance the performance of a thermoelectric device [12]. In fact, their quite general thermodynamic analysis invoking only the Onsager reciprocity relations and the positivity of entropy production suggested the possibility of a device delivering finite power while still reaching Carnot efficiency. Such a spectacular option deserves both further scrutiny and a search for a microscopic realization. In this Letter, we show that unitarity of the scattering matrix as a general physical principle imposes a strong restriction on the Onsager coefficients that lead to a significant reduction of the attainable efficiency of any thermoelectric device within the broad and well-established class of threeterminal models [13-16].

Thermoelectric transport can conveniently be discussed within the model sketched in Fig. 1. Two particle reservoirs of respective temperatures $T_{\mathrm{L}}>T_{\mathrm{R}}$ and chemical potentials $\mu_{\mathrm{L}}<\mu_{\mathrm{R}}$ are connected by a conductor $C$, which allows for the exchange of heat and particles. Consequently, as soon as the steady state is reached, constant heat and particle currents, $J_{q}$ and $J_{\rho}$, flow between the left and the right reservoirs. Depending on the sign of these currents, the machine works either as a power generator or a refrigerator. We now assume that both the temperature

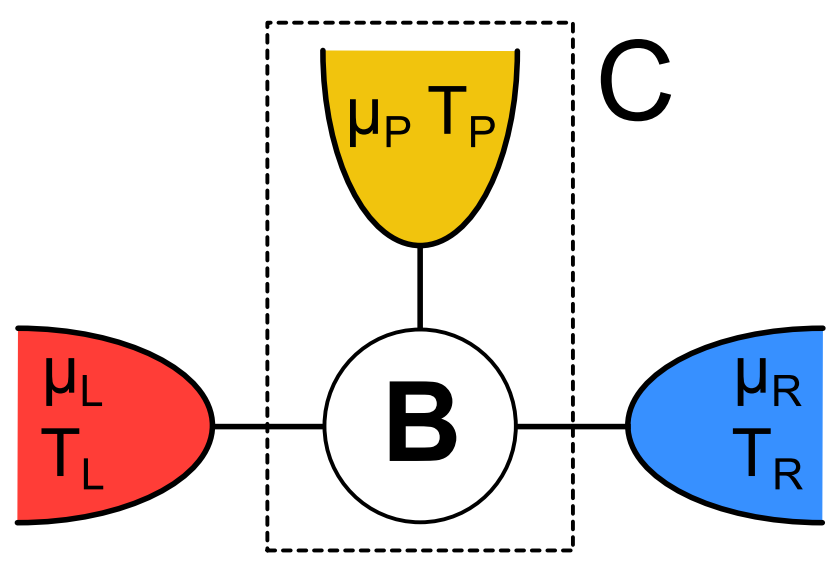

FIG. 1 (color online). Sketch of a thermoelectric device in the presence of a magnetic field $\mathbf{B}$. The entire dashed box represents the conductor $C$, which is connected to two reservoirs. For the special case of the three terminal model, the conductor consists essentially of a scattering region and an additional probe terminal. 
difference $\Delta T \equiv T_{\mathrm{L}}-T_{\mathrm{R}}>0$ and the chemical potential difference $\Delta \mu \equiv \mu_{\mathrm{L}}-\mu_{\mathrm{R}}<0$ are small compared to the respective reference values, which we choose to be $T \equiv T_{\mathrm{R}}$ and $\mu \equiv \mu_{\mathrm{R}}$. In this linear response regime, the currents $J_{\rho}$ and $J_{q}$ are related to the affinities $\mathcal{F}_{\rho} \equiv \Delta \mu / T$ and $\mathcal{F}_{q} \equiv \Delta T / T^{2}$ via the phenomenological equations

$J_{\rho}=L_{\rho \rho} \mathcal{F}_{\rho}+L_{\rho q} \mathcal{F}_{q} \quad J_{q}=L_{q \rho} \mathcal{F}_{\rho}+L_{q q} \mathcal{F}_{q}$.

The constant rate of entropy production accompanying this transport process reads

$$
\begin{aligned}
\dot{S} & =\mathcal{F}_{\rho} J_{\rho}+\mathcal{F}_{q} J_{q} \\
& =L_{\rho \rho} \mathcal{F}_{\rho}^{2}+L_{q q} \mathcal{F}_{q}^{2}+\left(L_{\rho q}+L_{q \rho}\right) \mathcal{F}_{\rho} \mathcal{F}_{q} .
\end{aligned}
$$

Clearly, the features of this machine are completely determined by the kinetic coefficients $L_{i j}(i, j=\rho, q)$, which are subject to two fundamental constraints. First, the second law requires $\dot{S} \geq 0$, which is equivalent to the conditions

$$
L_{\rho \rho}, L_{q q} \geq 0 \text { and } L_{\rho \rho} L_{q q}-\left(L_{\rho q}+L_{q \rho}\right)^{2} / 4 \geq 0 .
$$

Second, Onsager's theorem implies the symmetry relation

$$
L_{\rho q}(\mathbf{B})=L_{q \rho}(-\mathbf{B}) .
$$

Here, we have reintroduced the magnetic field $\mathbf{B}$ which was suppressed so far in order to keep the notation slim.

In the light of the Onsager reciprocity relation (4), the expression (1) for the total entropy production rate suggests a natural splitting of each current $J_{i}$ into a reversible and an irreversible part defined by

$$
J_{i}^{\mathrm{rev}} \equiv \frac{L_{i j}-L_{j i}}{2} \mathcal{F}_{j} \quad \text { and } \quad J_{i}^{\mathrm{irr}} \equiv L_{i i} \mathcal{F}_{i}+\frac{L_{i j}+L_{j i}}{2} \mathcal{F}_{j}
$$

Obviously, $J_{i}^{\text {rev }}$ vanishes for $\mathbf{B}=0$, i.e., for unbroken timereversal symmetry. However, once the latter is broken, although not contributing to $\dot{S}$, the reversible currents can, in principle, become arbitrarily large, since besides (3) no further general relations between $L_{i j}(\mathbf{B})$ and $L_{j i}(\mathbf{B})$ are known. Such unconstraint reversible currents could ultimately give rise to the possibility of dissipationless transport noticed by Benenti et al. [12] as mentioned above.

For progress investigating possible further constraints the phenomenological setup discussed so far must be made more specific. A paradigmatic model for the conductor $C$ is shown inside the dashed box in Fig. 1. It consists of a central scattering region, with a constant magnetic field $\mathbf{B}$, and a third electronic reservoir (terminal) $P$, whose temperature and chemical potential are chosen such that no net exchange of particles and heat with the rest of the system occurs. The scattering region is connected to all of the reservoirs via perfect, one-dimensional, infinitely long leads. In order to keep the model as simple as possible, we assume moderately damped [6], noninteracting electrons, which means that no inelastic scattering events take place outside the reservoirs and the electrons are transferred coherently between them. This setup constitutes the quite general class of three-terminal models on which we base our subsequent analysis. We emphasize that the additional terminal $P$ plays a crucial role since it is well known that in a purely coherent two terminal setup the offdiagonal Onsager coefficients must be even functions of the magnetic field [17] and hence there are no reversible currents. Such probe terminals, whose temperature and chemical potential are chosen self-consistently, were originally proposed by Büttiker [18] and have become a common tool to simulate inelastic events in an otherwise conservative system.

Since we now have to deal with three reservoirs, we need accordingly four affinities $\mathcal{F}_{\rho}^{A} \equiv\left(\mu_{A}-\mu\right) / T$ and $\mathcal{F}_{q}^{A} \equiv\left(T_{A}-T\right) / T^{2}(A=L, P)$, where we still use the temperature and chemical potential of the right reservoir as reference values $\mu$ and $T$. For convenience, we collect the affinities in two vectors $\mathcal{F}^{A} \equiv\left(\mathcal{F}_{\rho}^{A}, \mathcal{F}_{q}^{A}\right)$. Analogously, we define the current vectors $\mathbf{J}^{A}=\left(e J_{\rho}^{A}, J_{q}^{A}\right)$, with $J_{\rho}^{A}$ and $J_{q}^{A}$ the particle and heat currents flowing out of reservoir $A$ and $e$ the electronic unit charge. This vector notation allows us to write the phenomenological equations in the rather compact form

$$
\left(\begin{array}{c}
\mathbf{J}^{L} \\
\mathbf{J}^{P}
\end{array}\right)=\mathbb{L}^{\prime}\left(\begin{array}{c}
\mathcal{F}^{L} \\
\mathcal{F}^{P}
\end{array}\right) \text { with } \quad \mathbb{L}^{\prime} \equiv\left(\begin{array}{cc}
\mathbb{L}_{L L}^{\prime} & \mathbb{L}_{L P}^{\prime} \\
\mathbb{L}_{P L}^{\prime} & \mathbb{L}_{P P}^{\prime}
\end{array}\right) .
$$

Here, the $\mathbb{L}_{A B}^{\prime}(A, B=L, P)$ are $2 \times 2$-matrices of kinetic coefficients. By virtue of the additional constraint $\mathbf{J}^{P}=0$, we can moreover eliminate $\mathcal{F}^{P}$ and thereby reduce (6) to a system of two equations

$$
\mathbf{J}^{L}=\mathbb{L} \mathcal{F}^{L}
$$

with an effective matrix of kinetic coefficients

$$
\mathbb{L} \equiv \mathbb{L}_{L L}^{\prime}-\mathbb{L}_{L P}^{\prime} \mathbb{L}_{P P}^{\prime-1} \mathbb{L}_{P L}^{\prime} \equiv\left(\begin{array}{cc}
L_{\rho \rho} & L_{\rho q} \\
L_{q \rho} & L_{q q}
\end{array}\right)
$$

relevant for the net currents from $L$ to $R$.

Explicit expressions for the $2 \times 2$ block matrices $\mathbb{L}_{A B}^{\prime}$ of kinetic coefficients showing up in (6) arise from the multiterminal Landauer formula $[19,20]$

$$
\begin{aligned}
\mathbb{L}_{A B}^{\prime}= & \frac{e^{2} T}{h} \int_{-\infty}^{\infty} d E F(E)\left(\begin{array}{cc}
1 & \frac{E-\mu}{e} \\
\frac{E-\mu}{e} & \left(\frac{E-\mu}{e}\right)^{2}
\end{array}\right) \\
& \times\left(\delta_{A B}-\left|S_{A B}(E, \mathbf{B})\right|^{2}\right),
\end{aligned}
$$

where

$$
F(E) \equiv\left[4 k_{B} T \cosh ^{2}\left(\frac{E-\mu}{k_{B} T}\right)\right]^{-1}
$$

is the negative derivative of the Fermi function, $k_{B}$ denotes Boltzmann's constant and $h$ Planck's constant. 
$S_{A B}(E, \mathbf{B}) \neq S_{B A}(E, \mathbf{B})$ are the matrix elements of the $3 \times 3$ scattering matrix $\mathbb{S}(E, \mathbf{B})$ that describes the passage of electrons with energy $E$ through the scattering region. Current conservation requires that $\mathbb{S}(E, \mathbf{B})$ is unitary and the time reversal invariance of unitary dynamics implies the symmetry $\mathbb{S}(E, \mathbf{B})=\mathbb{S}(E,-\mathbf{B})^{t}[19]$. We emphasize that in the presence of a magnetic field, this symmetry permits $\mathbb{S}(E, \mathbf{B})$ to be nonsymmetric. Consequently, despite the fact that the $\mathbb{L}_{A B}^{\prime}$ are still symmetric, the reduced matrix (8) will generically acquire a nonvanishing asymmetric part as desired.

We now derive a constraint on the asymmetry of the reduced matrix $\mathbb{L}$. First, we define the Hermitian matrices

$$
\mathbb{K}^{\prime} \equiv\left(\mathbb{L}^{\prime}+\mathbb{L}^{\prime t}\right)+i \sqrt{3}\left(\mathbb{L}^{\prime}-\mathbb{L}^{\prime t}\right)
$$

and

$$
\mathbb{K} \equiv\left(\mathbb{L}+\mathbb{L}^{t}\right)+i \sqrt{3}\left(\mathbb{L}-\mathbb{L}^{t}\right) \equiv\left(\begin{array}{cc}
K_{\rho \rho} & K_{\rho q} \\
K_{\rho q}^{*} & K_{q q}
\end{array}\right),
$$

where $\mathbb{L}^{\prime}$ is the full $4 \times 4$ Onsager-matrix introduced in (6), while $\mathbb{L}$ is the reduced matrix (8). Second, as a consequence of the unitarity of the scattering matrix $\mathbb{S}(E, \mathbf{B})$, $\mathbb{K}^{\prime}$ can be shown to be positive semidefinite on $\mathbb{C}^{4}$ [21]. Third, since for any $\mathbf{z} \in \mathbb{C}^{2}$

$\mathbf{z}^{\dagger} \llbracket \mathbb{z}=\mathbf{z}^{\prime \dagger} \mathbb{K}^{\prime} \mathbf{z}^{\prime} \geq 0 \quad$ with $\quad \mathbf{z}^{\prime} \equiv\left(\begin{array}{c}\mathbf{z} \\ -\mathbb{L}_{P P}^{\prime-1} \mathbb{L}_{P L}^{\prime} \mathbf{z}\end{array}\right)$,

it then follows that the reduced matrix $\mathbb{K}$ must be positive semidefinite on $\mathbb{C}^{2}$. Therefore the matrix elements of $\mathbb{K}$ have to obey the inequalities

$$
K_{\rho \rho}, K_{q q} \geq 0 \quad \text { and } \quad K_{\rho \rho} K_{q q}-K_{\rho q} K_{\rho q}^{*} \geq 0 .
$$

We note that the $K_{i j}(i, j=\rho, q)$ are rather complicated functions of the matrix elements of the full Onsager-matrix $\mathbb{L}^{\prime}$, which would make it a quite challenging task to obtain (14) directly from (8) and (9). If we insert the definition (12) of the $K_{i j}$ in terms of the $L_{i j}$, we immediately get the relation

$$
L_{\rho \rho} L_{q q}+L_{\rho q} L_{q \rho}-L_{\rho q}^{2}-L_{q \rho}^{2} \geq 0,
$$

which constitutes our first main result. We emphasize that (15) provides a much stronger constraint than (3) since the former can be rewritten as

$$
L_{\rho \rho} L_{q q}-\left(L_{\rho q}+L_{q \rho}\right)^{2} / 4 \geq 3\left(L_{\rho q}-L_{q \rho}\right)^{2} / 4 .
$$

Indeed, we recover (3) only if $L_{\rho q}$ is equal to $L_{q \rho}$. As soon as the kinetic coefficients contain finite asymmetric parts, the left hand side of (15) must be strictly larger than zero. In other words, the reversible currents associated with the asymmetric part of $\mathbb{L}$ come at the price of a stronger lower bound on the Onsager coefficients and hence on the entropy production rate (2) than the bare second law (3) requires.
The new bound (15) has profound consequences for the performance of the model as a thermoelectric heat engine. Following the lines of Benenti et al. [12], we introduce the dimensionless parameters

$$
y \equiv L_{\rho q} L_{q \rho} / \text { Det } \mathbb{L} \quad \text { and } \quad x \equiv L_{\rho q} / L_{q \rho} .
$$

Expressed in terms of $x$ and $y$, the inequality (15) reads

$h(x) \leq 4 y \leq 0 \quad$ if $x<0, \quad 0 \leq 4 y \leq h(x) \quad$ if $x>0$,

where $h(x) \equiv 4 x /(x-1)^{2}$. The most important benchmarks for the performance of a heat engine-namely, the maximum efficiency $\eta_{\max }$ and the efficiency at maximum power $\eta\left(P_{\max }\right)$ - admit the analytic expressions [12]

$$
\eta_{\max }=\eta_{C} x \frac{\sqrt{y+1}-1}{\sqrt{y+1}+1} \quad \text { and } \quad \eta\left(P_{\max }\right)=\eta_{C} \frac{x y}{4+2 y} .
$$

Here, we have denoted by $\eta_{C}=1-T_{\mathrm{R}} / T_{\mathrm{L}} \approx T \mathcal{F}_{q}^{L}$ the Carnot efficiency, which is the absolute upper bound for the attainable efficiency following from the second law. Both benchmarks become maximal for $4 y=h(x)$. The resulting bounds, $\eta_{\max }^{*}$ and $\eta^{*}\left(P_{\max }\right)$, which are our second main result, are plotted in Fig. 2. It shows how the maximum efficiency decays rapidly as the asymmetry parameter $x$ deviates from its symmetric value 1 . In the limit $x \rightarrow \pm \infty$, maximum efficiency and efficiency at maximum power both approach $\eta_{C} / 4$. This result implies essentially that from the perspective of maximally attainable efficiency the thermodynamic cost of the reversible currents is larger than the benefit they bring. For efficiency at maximum power, the situation is somewhat different. If $x$ is only slightly larger than 1 , the Curzon-Ahlborn limit $\eta_{C A}=\eta_{C} / 2$ [22-26], reached for $x=1$, can be overcome in a small range of $x$ values with a maximum of $4 \eta_{C} / 7$ at $x=4 / 3$. This result shows that despite the strong bounds on $\eta_{\max }$ it may in principle be possible to improve the performance of the machine by breaking the time-reversal symmetry slightly.

Finally, we discuss the consequence of the bound (15) for the model as a refrigerator. In this case the most important benchmark is the coefficient of performance $\varepsilon \equiv-J_{q}^{L} / T \mathcal{F}_{\rho}^{L} J_{\rho}^{L}[2]$, defined as the ratio of the heat current extracted from the cold reservoir and the absorbed power. In terms of the dimensionless parameters (17) $\varepsilon$ reads [12]

$$
\varepsilon=\frac{\eta_{C}^{r}}{x} \frac{\sqrt{y+1}-1}{\sqrt{y+1}+1}
$$

where $\eta_{C}^{r}=T_{\mathrm{R}} /\left(T_{\mathrm{L}}-T_{\mathrm{R}}\right) \approx 1 / T \mathcal{F}_{q}^{L}$ is the efficiency of an ideal refrigerator. Like for $\eta_{\max }$ and $\eta\left(P_{\max }\right)$, the maximum $\varepsilon^{*}$ of $\varepsilon$ is attained for $4 y=h(x)$. The resulting bound is plotted in Fig. 3. Again, we observe that the efficiency deteriorates rapidly as $x$ deviates from 1 . 

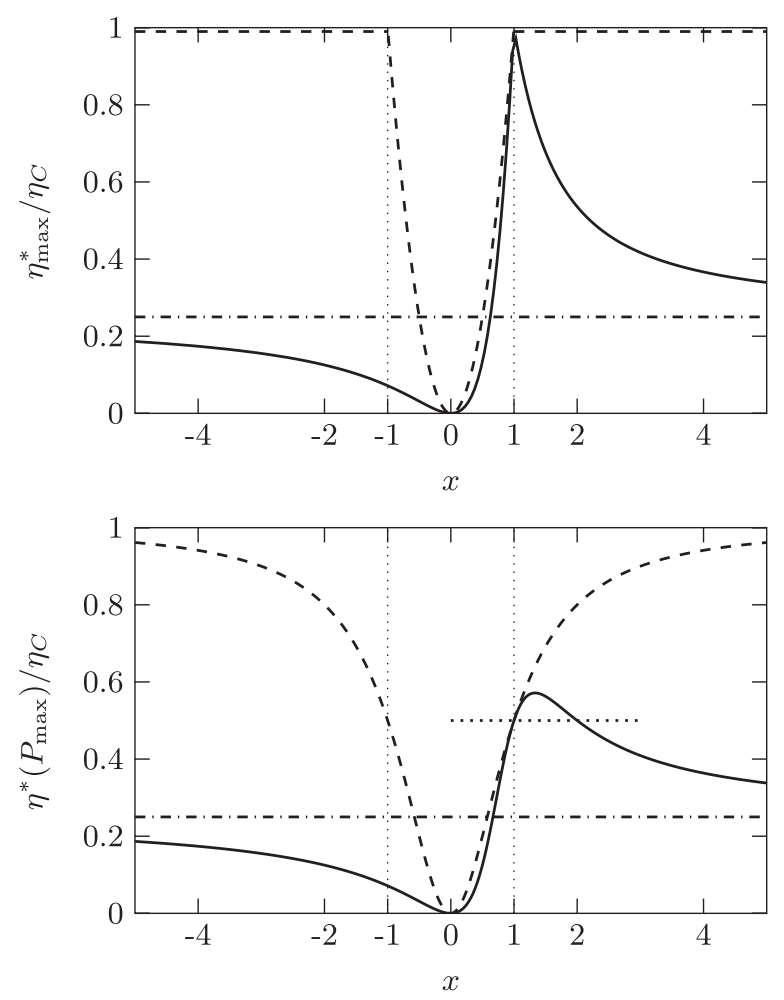

FIG. 2. Bounds on benchmarks in units of $\eta_{C}$ as functions of the asymmetry parameter $x$. The upper diagram shows $\eta_{\max }^{*}$, the lower one $\eta\left(P_{\max }\right)^{*}$. The solid curves represent the bounds following from relation (15). In the limit $x \rightarrow \pm \infty$ both functions asymptotically approach the value $1 / 4$, which is shown by the dash-dotted lines. For comparison, the bounds obtained by Benenti et al. [12] solely from the second law (3) have been included as dashed curves. The dotted line in the lower panel indicates the Curzon-Ahlborn limit 1/2.

In conclusion, we have investigated the thermoelectric transport properties of the most general version of the paradigmatic three terminal model in the presence of broken time reversal symmetry. We have derived a strong

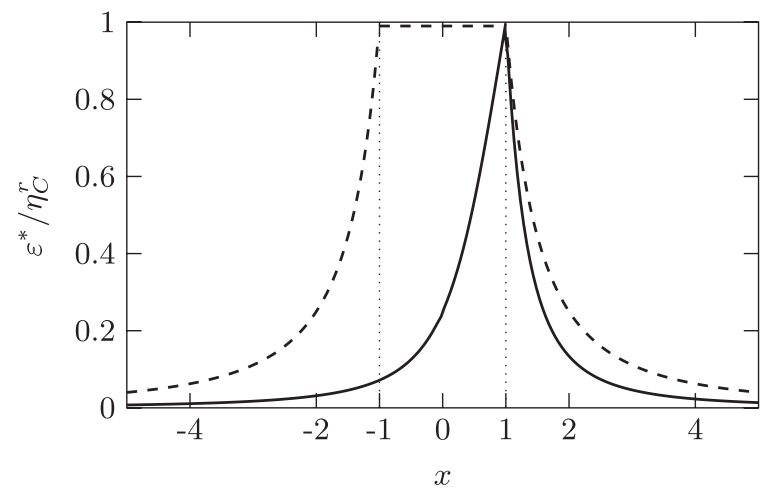

FIG. 3. Bounds on the coefficient of performance of a refrigerator $\varepsilon$ in units of $\eta_{C}^{r}$ as a function of the asymmetry parameter $x$. The dashed line follows from bare second law, the solid line from the stronger relation (15). constraint on the linear transport coefficients that can be obtained neither from Onsager's principle of microreversibility nor from thermodynamic arguments invoking only the second law. This constraint implies strong bounds on both maximum efficiency and efficiency at maximum power if this device is operated as a thermoelectric heat engine or refrigerator.

We emphasize that our results rely solely on the unitarity of the scattering matrix. Therefore our new relation (15) applies to any specific model which can be formulated within the three terminal setup, including especially the ones based on quantum dots [16] and ballistic microjunctions [15], which have been proposed in this context recently. It might even apply to a model introduced by Entin-Wohlman and Aharony [14], which includes a phonon bath locally interacting with electrons transferred between the reservoirs. Furthermore, it is noteworthy that by following exactly the same lines the constraint (15) can be shown to apply to the three terminal railway switch transport model [13]. This model may be regarded as a classical analogue of the quantum three terminal model discussed here. Indeed, our considerations are not restricted to the quantum realm, since ultimately the unitarity of the scattering matrix is nothing but the manifestation of the law of current conservation, which should be considered the fundamental principle underlying our bound.

Despite this generality, the question whether a similar bound can be found for more complex model classes, including for example a larger number of probe terminals or models with genuinely interacting electrons, remains open and should constitute an important subject for further investigations.

K. B. and U.S. acknowledge support from ESF through the EPSD network. K.S. acknowledges support from MEXT (23740289).

[1] L. Onsager, Phys. Rev. 38, 2265 (1931).

[2] H. B. Callen, Thermodynamics and an Introduction to Thermostatics (John Wiley \& Sons, New York, 1985), 2nd ed.

[3] H. B. G. Casimir, Rev. Mod. Phys. 17, 343 (1945).

[4] C. Goupil, W. Seifert, K. Zabrocki, E. Müller and G. J. Snyder, Entropy 13, 1481 (2011).

[5] G. D. Mahan and J. O. Sofo, Proc. Natl. Acad. Sci. U.S.A. 93, 7436 (1996).

[6] T.E. Humphrey, R. Newbury, R. P. Taylor and H. Linke, Phys. Rev. Lett. 89, 116801 (2002).

[7] T. E. Humphrey and H. Linke, Phys. Rev. Lett. 94, 096601 (2005).

[8] C. J. Vineis, A. Shakouri, A. Majumdar, and M. G. Kanatzidis, Adv. Mater. 22, 3970 (2010).

[9] L. E. Bell, Science 321, 1457 (2008).

[10] G. J. Snyder and S. Toberer, Nat. Mater. 7, 105 (2008).

[11] M. S. Dresselhaus, G. Chen, M. Y. Tang, R. G. Yang, H. Lee, D. Z. Wang, Z. F. Ren, J.-P. Fleurial, and P. Gogna, Adv. Mater. 19, 1043 (2007). 
[12] G. Benenti, K. Saito and G. Casati, Phys. Rev. Lett. 106, 230602 (2011).

[13] M. Horvat, T. Prosen, G. Benenti and G. Casati, Phys. Rev. E 86, 052102 (2012).

[14] O. Entin-Wohlman and A. Aharony, Phys. Rev. B 85, 085401 (2012).

[15] D. Sánchez and L. Serra, Phys. Rev. B 84, 201307(R) (2011).

[16] K. Saito, G. Benenti, G. Casati and T. Prosen, Phys. Rev. B 84, 201306(R) (2011).

[17] M. Büttiker, IBM J. Res. Dev. 32, 317 (1988).

[18] M. Büttiker, Phys. Rev. B 33, 3020 (1986).
[19] P. N. Butcher, J. Phys. Condens. Matter 2, 4869 (1990).

[20] U. Sivan and Y. Imry, Phys. Rev. B 33, 551 (1986).

[21] See Supplemental Material at http://link.aps.org/ supplemental/10.1103/PhysRevLett.110.070603 for a proof.

[22] F. L. Curzon and B. Ahlborn, Am. J. Phys. 43, 22 (1975).

[23] C. Van den Broeck, Phys. Rev. Lett. 95, 190602 (2005).

[24] M. Esposito, R. Kawai, K. Lindenberg and C. Van den Broeck, Phys. Rev. Lett. 105, 150603 (2010).

[25] U. Seifert, Phys. Rev. Lett. 106, 020601 (2011).

[26] C. Van den Broeck, N. Kumar and K. Lindenberg, Phys. Rev. Lett. 108, 210602 (2012). 